\title{
Genetic variants in RORA are associated with asthma and allergy markers in an admixed population
}

\author{
Louise Correia Lima ${ }^{\mathrm{a}}$, Gerson de Almeida Queiroz ${ }^{\mathrm{b}}$, Ryan dos Santos Costa ${ }^{\mathrm{b}}$, \\ Neuza Maria Alcantara-Neves ${ }^{\mathrm{b}}$, Cíntia Rodrigues Marques ${ }^{\mathrm{c}}$, Gustavo Nunes de Oliveira Costa ${ }^{\mathrm{d}}$, \\ Mauricio Lima Barreto $^{\mathrm{d}}$, Camila Alexandrina Viana Figueiredo ${ }^{\mathrm{b}}$, Valdirene Leão Carneiro ${ }^{\mathrm{a}, *}$ \\ a Departamento de Ciências da Vida, Universidade do Estado da Bahia, Salvador, BA, Brazil \\ ${ }^{\mathrm{b}}$ Instituto de Ciências da Saúde, Universidade Federal da Bahia, Salvador, BA, Brazil \\ ${ }^{\mathrm{c}}$ Instituto Multidisciplinar em Saúde, Universidade Federal da Bahia, Salvador, BA, Brazil \\ ${ }^{\mathrm{d}}$ Instituto de Saúde Coletiva, Universidade Federal da Bahia, Salvador, BA, Brazil
}

\section{A R T I C L E I N F O}

\section{Keywords:}

Polymorphism

Atopic sensitization

RORA

Asthma

\begin{abstract}
A B S T R A C T
Asthma and allergy affect hundreds of millions of people from childhood to old age. In most of them, the inflammatory process of respiratory allergies involves the participation of type 2 cytokines, derived from $\mathrm{T}$ helper-2 (Th2)-cell, and Group 2 Innate Lymphoid (ILC2) Cells. An efficient memory Th2 cell response is dependent on IL-13 produced by ILC2s, causing allergic lung inflammation and elevated serum levels of immunoglobulin E. ILC2 cells are derived from common lymphoid progenitors and their growing depends on the transcription factor RORA. The aim of this work was to identify genetic variants in RORA associated with asthma phenotypes and allergy markers. Genomic DNA samples of 1246 individuals participating from Social Changes Asthma and Allergy in Latin America Program (SCAALA) have been genotyped using Illumina Human 2.5 Omni Beadchip. Logistics regressions have been performed to analyze the association among RORA variants and asthma, skin prick tests (SPT), specific IgE and type 2 cytokine production. Twelve single nucleotide variants (SNVs) were significantly associated with atopy $(\mathrm{P}<0.01)$, in which four of them, rs10162630, rs17191519, rs17270243, and rs55796775 and their haplotypes were strongly and positively associated $(\mathrm{P}<0.001)$. Furthermore, these variants increased the RORA gene expression in silico analysis. Other SNVs in RORA were associated with allergy markers, atopic and non-atopic asthma. Therefore, it is believed that variants in RORA gene may influence immunologic features of asthma and allergies and could be possible targets for future treatment of allergic diseases.
\end{abstract}

\section{Introduction}

The major chronic respiratory diseases include asthma and allergic rhinitis, which affect hundreds of millions individuals worldwide. The frequency of these diseases has caused prevalence rates to increase in both developed and developing countries [1]. Clinical asthma features include wheezing, mucus production, airflow limitation and bronchial hyperreactivity to environmental stimuli [2]. Among several asthma phenotypes, two are very well characterized: (1) atopic asthma, defined by positive serum specific Immunoglobulin E (IgE) antibodies and/or skin prick test (SPT) positive to any tested allergen; (2) non-atopic asthma, with absence of allergen-specific serum IgE and/or SPT and is associated with air pollution, including ozone, cigarette smoke and diesel particulates, and higher frequency in adult women [3].

The inflammatory process of respiratory allergies involves interactions and cooperation of many cell types, causing a type 2 immune response $[4,5]$ mediated by eosinophils, mast cells, basophils, Th2 cells, Group 2 Innate Lymphoid (ILC2) cells and IgE-producing B cells. Th2 cells are sources of type 2 cytokines, such as IL-4, IL-5, IL-9 and IL-13. These cytokines are responsible for unleashing a cascade of events, such as IgE-triggered hypersensitivity to aeroallergens, activation and migration of eosinophils, differentiation for a Th2 immune response, bronchial hyperreactivity and metaplasia of globet cells with mucus production in the epithelium and mucosal subepithelium of the airways. However, for some time asthma is no longer considered as pathology of only Th2 profile, other cell types participate in the

Abbreviations: B. germanica, Blattella germanica; B. tropicalis, Blomia tropicalis; D. pteronyssinus, Dermatophagoides pteronyssinus; GWAS, Genome-Wide Association Studies; ILC2, Group 2 Innate Lymphoid; LD, linkage disequilibrium; Periplaneta americana, P. americana; sIgE, specific Immunoglobulin E; SPT, skin prick tests; SNV, single nucleotide variant

* Corresponding author at: Departamento de Ciências da Vida, Universidade do Estado da Bahia, Rua Silveira Martins, 248, 40301-110 Cabula, Salvador, BA, Brazil.

E-mail address: vcarneiro@uneb.br (V.L. Carneiro). 
pathophysiology of asthma and allergic diseases such as ILC2 cells [6]. There is also the participation of Th17 cells, responsible for the neutrophilic inflammatory profile of asthma in some patients. ILC2 cells do not have specific antigenic receptors, but like Th2 cells, they respond to the stimulus by triggering IL-25, IL-33, and Thymic Stromal Lymphopoietin (TSLP), producing IL-5, IL -9 and IL-13. ILC2-derived IL-13 mounts an efficient memory Th2 cell response to allergens, causing allergic lung inflammation and elevated serum levels of IgE $[7,8]$. ILC2 cells are derived from lymphoid progenitors common in response to IL7 and IL-33 and their growing depend on the transcription factors Zinc Finger 3 (GATA-3) and Retinoic acid-dependent Orphan Receptor Alpha (RORA) [9-11].

Retinoic acid-dependent Orphan Receptors (RORs) are members of the Nuclear Receptor protein superfamily and comprise three members: ROR-alpha, ROR-beta and ROR-gamma. RORA has four variants, called RORA-1, RORA-2, RORA-3 and RORA-4, which differ in the aminoterminal region, as well as gene expression in different tissues $[12,13]$. In addition, RORA, as well as the other nuclear receptors, shares a common modular structure composed of several domains: the aminoterminal domain, the DNA Binding Domain (DBD), the Ligand Binding Domain (LBD) and a flexible domain that connects the DBD and LBD. In RORA, the DNA binding domain is named RORE $[9,14]$. RORA regulates gene transcription through monomer binding to the RORE response element. When it interacts with coactivators and corepressors, RORA can induce or suppress, respectively, the transcription of target genes [15]. The main known functions of RORA are: circadian rhythm regulator, participation in the metabolism and development of the immune system $[5,16]$. Experimental models of airway inflammation have shown that mice deficient in the RORA gene have attenuated immune system cell infiltration in the lung, decreased pulmonary mucosal hyperplasia, reduced Th2 cells, ILC2 cells, IL-4, IL-5 and IL-13 cytokines (Fig. 1). Other papers suggest a role for RORA in the regulation of Th2 inflammation occurring in the lung, as well as a risk factor for asthma in European Americans [13,17].

Asthma is a complex disease and genetically heterogeneous; the interaction between environmental and genetic factors leads to the occurrence of allergic respiratory diseases $[18,19]$. Genome-Wide Association Studies (GWAS) have demonstrated association between genetic variants in RORA and asthma. Moreover, other GWAS have described genes, such as IL33, IL13 and STAT6 which, like RORA, are molecular components of the pathophysiology of asthma [20-23]. Acevedo et al. [24] have demonstrated that genetic variants in RORA are risk factors for childhood asthma and have epistasis with polymorphisms in NPSR1. The NPS/NPSR1 pathway has biological interactions with RORA and other circadian clock genes that may have effects on the rhythmic occurrence of asthma symptoms.

Studies suggest that orphan nuclear receptors are an active field of research because of its potential for binding to ligands, which can be used to modulate these receptors in order to develop targeted therapies for various diseases $[9,13]$. It is known that RORA is constitutively active, presenting greater transcriptional activity when binding to a coactivator. However, there is the possibility of RORA binding to an inverse agonist, may allow suppression of transcription. Studying genetic variants in RORA can contribute to the identification of the pathogenic asthma mechanisms that are not still well elucidated and thus, discover new therapeutic options. In this context, the aim of this work was to identify genetic variants in RORA associated with asthma phenotypes and atopy markers in an admixed population of individuals from Salvador, Bahia, Brazil.

\section{Material and methods}

\subsection{Study population and data collection}

Data collected from 1246 children, included in the SCAALA (Social Changes Asthma and Allergy in Latin America) cohort, were analyzed. SCAALA was created to study the effect of a sanitation program on the children's health (age 4 to 11 years) in the city of Salvador, located in the Northeast of Brazil, from 1996 to 2004. They were again evaluated in 2005 , with the application of new questionnaires for asthma diagnosis. The methods applied in this study were reported in previous studies [25-27].

Ethical approval was obtained by the National Research Ethics Committee 120.616. The written, free and informed consent was appropriately obtained from parents or legal guardian of each child.

\subsection{Definition of asthma and atopy}

Using the ISAAC questionnaire phase II (International Study of Asthma and Allergies in Childhood), children were classified as having asthma if their parents or guardians reported wheezing in the last 12 months and at least one of the following: clinical diagnosis of asthma, wheezing with exercise in the last 12 months, four or more episodes of wheezing in the last 12 months and waking up at night because of wheezing in the last 12 months [26].

Atopy was defined as the presence of at least one anti-allergen IgE test with serum levels equal to or greater than $0.70 \mathrm{kU} / \mathrm{L}$, regardless of results of SPT [25].

The skin prick test was performed on the right forearm of each child using standardized extracts (ALK-Abelló, São Paulo, Brazil) for four

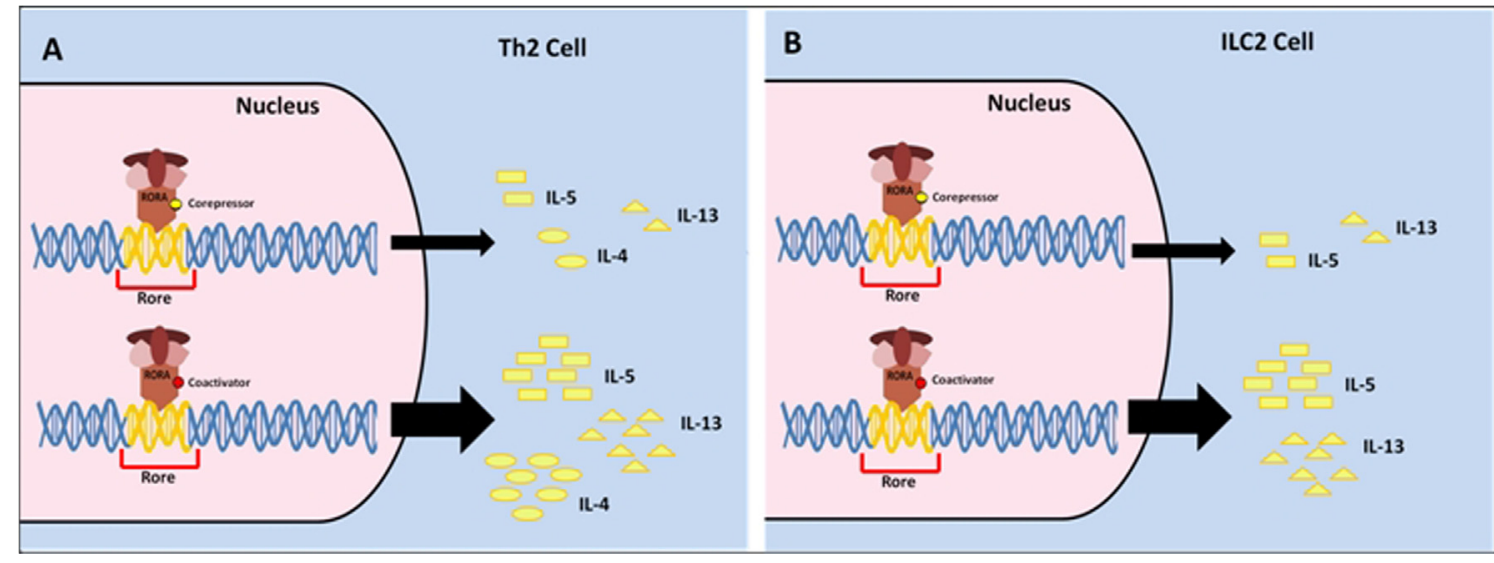

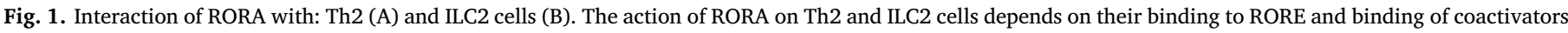

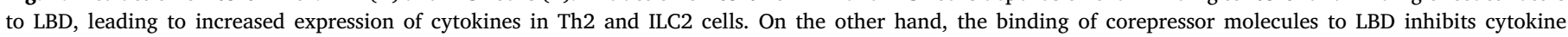

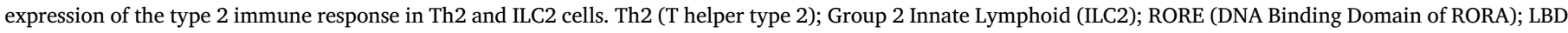
(Ligand Binding Domain). 
allergens: Blattella germanica, Blomia tropicalis, Dermatophagoides pteronyssinus and Periplaneta americana. These tests were previously described by Barreto et al. [26]. Saline solution was used for negative control and $10 \mathrm{mg} / \mathrm{mL}$ of histamine for positive control. The reaction to each allergen was read after $15 \mathrm{~min}$ and considered positive if the papule diameter was at least $3 \mathrm{~mm}$ larger when compared to the negative control.

Each child's blood was collected in a heparinized tube and the serum kept frozen until use. The Pharmacia Immuno CAP System IgE FEIA (Pharmacia, Uppsala, Sweden) was used to measure the serum levels of IgE anti-mite (Dermatophagoides pteronyssinus and Blomia. tropicalis) and anti-cockroach (Periplaneta americana and Blatella germanica) in the subjects' blood, according to the manufacturer's instructions. Atopic condition was considered when serum IgE levels were greater than or equal to $0.70 \mathrm{kU} / \mathrm{L}$ for at least one tested allergen.

Whole blood was cultured in a $5 \% \mathrm{CO}_{2}$ atmosphere during 5 days for the detection of IL-13 and IL-5. These cytokines were detected in culture supernatant by the capture ELISA method, according to the manufacturer's instructions (Pharmigen, San Diego, CA, USA) [25].

\subsection{DNA extraction and genotyping}

DNA extraction was performed from the peripheral blood samples of 1246 SCAALA subjects, according to the protocols of QIAGEN kit (Gentra Puregene Blood Kit, Hilden, Germany). All samples to be genotyped were standardized at a concentration of $50 \mathrm{ng} / \mu \mathrm{L}$ and identified in bar code tubes. The samples were genotyped using the Illumina Human Omni 2.5-8 Bead Chip Kit, which consists of a large platform with approximately 2.5 million markers of the human genome (www. ilummina.com). For this study, the RORA genetic information was extracted between positions 60781040 and 61517218 on chromosome 15.

\subsection{Statistical analysis}

Using Plink program (version 1.07), the single nucleotide variants (SNVs) were filtered according to the following criteria: HardyWeinberg equilibrium test ( $\mathrm{P}$-value $<0.001)$; minor allele frequency ( $>0.1$ ) and percentage of loci lost (MIND > 0.1 and GENO > 0.1). The association analyses were adjusted for sex, age, helminth infection and major components (PC1 and PC2) to correct confounding factors from the population structure $[20,28]$.

The analyses were performed under an additive model and the Odds Ratio (OR) was estimated, as well as 1000 permutations procedures was used to estimate the statistical significance of multiple correlation tests in the genetic association analysis [29,30]. Results obtained from this analysis having a confidence interval of $99.0 \%$ and a P-value less than 0.01 were considered statistically significant.

The haplotype analysis was performed in the SNPStats version 1.22.0 program (www.rdocumentation.org).

The Mann-Whitney test was used to compare IL-5 and IL-13 production levels among different rs10162630, rs17191519, rs17270243 and rs55796775 genotypes. Statistical significance was considered with an alpha error of $5 \%$. These statistical analyses were performed using Prism software version 5 (GraphPad Inc., San Diego, CA).

The linkage disequilibrium (LD) analysis was carried out for each selected SNV, which refers to the association of alleles on the same chromosome but at different loci. Haploview 4.2 software was used to calculate the degree of confidence in the D́ value [31].

\subsection{In silico analysis}

The possible role of each SNV was obtained through information available from the National Biotechnology Information Center (NCBI) (www.ncbi.nlm.nih.gov).

Each SNV was also scored according to the scale of the RegulomeDB project (regulomedb.org), which consists of a database for the interpretation of regulatory variants in the human genome. RegulomeDB identifies probable functions of functional genetic variants and regulatory mechanisms through computational predictions and manual annotations. Data sources are combined in a tool that scores functional variants and provides testable hypotheses regarding their function [32]. The authors of the RegulomeDB project have developed a heuristic scoring system based on the functional reliability of a variant. The scoring system represents, on a growing scale of confidence, the functional location of a variant and its probable functional consequence. Scores from $1 \mathrm{a}$ to $1 \mathrm{f}$ indicate that the variant is likely to affect binding and is linked to the expression of a target gene, and the scores from 2a to $2 \mathrm{c}$ only indicate that the variant is likely to affect binding. While scores from $3 \mathrm{a}$ and $3 \mathrm{~b}$ indicate that variants are less likely to affect binding, and scores of 4,5 , and 6 indicate minimal binding evidence or no data are available [32].

All SNVs were subjected to an analysis on the GTEx portal (www. gtexportal.org), from a consortium (Consortium, 2015), created by the National Institutes of Health Common Fund (Genotype-Tissue Expression Project). This consortium provides resources that enable the study of human gene expression and regulation and its relation to genetic variation and other molecular phenotypes in various human tissue types. The project authors analyzed the expression of global RNA in individual tissues and the levels of gene expression were treated as quantitative traits. Therefore, variations in gene expression that are highly correlated with genetic variation can be identified as expression of quantitative trait loci, named eQTLs.

\section{Results}

\subsection{Study population}

Table 1 summarizes the characteristics of the study population. It

Table 1

Characteristics of the SCAALA population according to asthma status and the variables included in the study.

\begin{tabular}{|c|c|c|c|c|c|}
\hline \multirow[t]{2}{*}{ Variables } & \multicolumn{5}{|c|}{ Subject group (1246) } \\
\hline & Nonasthmatic & $\%$ & Asthmatic & $\%$ & P-value \\
\hline & $942^{*}$ & $75.9 \%$ & $274^{*}$ & $22 \%$ & \\
\hline \multicolumn{6}{|l|}{ Age } \\
\hline$\leq 5$ & 314 & $33.3 \%$ & 132 & $48.2 \%$ & \\
\hline $6-7$ & 337 & $35.8 \%$ & 88 & $32.1 \%$ & 0.000 \\
\hline$\geq 8$ & 291 & $30.9 \%$ & 54 & $19.7 \%$ & \\
\hline \multicolumn{6}{|l|}{ Sex } \\
\hline Male & 506 & $53.7 \%$ & 151 & $55.1 \%$ & 0.684 \\
\hline Female & 436 & $46.3 \%$ & 123 & $44.9 \%$ & \\
\hline \multicolumn{6}{|l|}{ Specific IgE for } \\
\hline Blomia tropicalis & 288 & $30.6 \%$ & 128 & $46.7 \%$ & 0.000 \\
\hline $\begin{array}{c}\text { Dermatophagoides } \\
\text { pteronyssinus }\end{array}$ & 179 & $19 \%$ & 91 & $33.2 \%$ & 0.000 \\
\hline Periplaneta americana & 81 & $8.6 \%$ & 31 & $11.3 \%$ & 0.171 \\
\hline Blatella germanica & 117 & $12.4 \%$ & 47 & $17.2 \%$ & 0.044 \\
\hline \multicolumn{6}{|l|}{ Skin Prick test for } \\
\hline Blomia tropicalis & 192 & $20.4 \%$ & 76 & $27.7 \%$ & 0.010 \\
\hline $\begin{array}{c}\text { Dermatophagoides } \\
\text { pteronyssinus }\end{array}$ & 131 & $13.9 \%$ & 62 & $22.6 \%$ & 0.001 \\
\hline Periplaneta americana & 120 & $12.7 \%$ & 49 & $17.9 \%$ & 0.030 \\
\hline Blatella germanica & 70 & $7.4 \%$ & 31 & $11.3 \%$ & 0.040 \\
\hline $\begin{array}{l}\text { IL-13 production by } \\
\text { Dermatophagoides. } \\
\text { pteronyssinus } \\
\text { stimulus }^{\mathrm{a}}\end{array}$ & 174 & $18.5 \%$ & 52 & $19 \%$ & 0.907 \\
\hline
\end{tabular}

Data were analyzed using the chi-squared test.

* 30 subjects presented data miss and were excluded to analysis.

a Response of asthmatic and nonasthmatic individuals from the lowest detection point for each cytokine in cells stimulated cultures. 
Table 2

Genetics information of main SNVs associated with asthma and allergy markers in this study.

\begin{tabular}{|c|c|c|c|c|c|}
\hline SNV & $\begin{array}{l}\text { Location } \\
\text { Allele }^{\mathrm{a}}\end{array}$ & $\mathrm{MAF}^{\mathrm{b}}$ & $\mathrm{HWE}^{\mathrm{c}}$ & Function $^{\mathrm{d}}$ & $\begin{array}{l}\text { RegulomeDB } \\
\text { score }\end{array}$ \\
\hline rs10162630 & $\mathrm{A} / \mathrm{G}$ & 0.45 & 0.73 & Intron variant & 5 \\
\hline rs11071584 & $\mathrm{A} / \mathrm{C}$ & 0.31 & 0.64 & Intron variant & 5 \\
\hline rs12903172 & $\mathrm{C} / \mathrm{T}$ & 0.25 & 0.26 & Intron variant & 5 \\
\hline rs17191519 & $\mathrm{G} / \mathrm{A}$ & 0.10 & 0.65 & Intron variant & $3 a$ \\
\hline rs17270243 & $\mathrm{G} / \mathrm{A}$ & 0.17 & 0.28 & Intron variant & 6 \\
\hline rs2414681 & $\mathrm{A} / \mathrm{C}$ & 0.31 & 0.59 & $\begin{array}{l}\text { intron variant, nc } \\
\text { transcript variant }\end{array}$ & 6 \\
\hline rs34720147 & $\mathrm{T} / \mathrm{C}$ & 0.26 & 0.30 & Intron variant & -7 \\
\hline rs4775301 & $\mathrm{C} / \mathrm{T}$ & 0.29 & 0.44 & Intron variant & 4 \\
\hline rs55796775 & $\mathrm{T} / \mathrm{G}$ & 0.40 & 0.52 & Intron variant & 6 \\
\hline rs7169281 & $\mathrm{C} / \mathrm{T}$ & 0.31 & 0.55 & Intron variant & 5 \\
\hline rs726914 & $\mathrm{A} / \mathrm{G}$ & 0.19 & 0.45 & Intron variant & 5 \\
\hline rs8024133 & $\mathrm{T} / \mathrm{C}$ & 0.30 & 0.95 & Intron variant & 4 \\
\hline
\end{tabular}

${ }^{\text {a }}$ First is alternative allele and the second is reference allele $(1 / 2)$.

b MAF: Minor Allele Frequency.

c HWE: Hardy-Weinberg Equilibrium.

d NCBI (National Center for Biotechnology Information).

was observed different proportions of asthmatic subjects across all age groups $(\mathrm{P}<0.01)$. Differences in markers of allergy, such as SPT and specific IgE levels were statistically significant $(P<0.05)$ between non-asthmatic and asthmatic groups, being all of them greater among asthmatics.

\subsection{Description of variants in RORA gene}

For the accomplishment of this study, 824 SNVs from the Illumina genotyping chip belonging to the RORA were extracted. 178 SNVs were excluded by the frequency test (MAF $<0.10$ ). No SNV was excluded because of poor genotyping (MIND $>0.1$ ), missingness (GENO $>0.1$ ) or by the Hardy-Weinberg Equilibrium (HWE) test $(P \leq 0.001)$. Thus, 646 SNVs were included in the association analysis.

Table 2 contains information on the top- 12 SNVs, in accordance with the statistical criteria established in Section 2.4 and which were associated with two or more outcomes. It also shows a MAF ranging between 0.10 and 0.45 . The complete list of all SNVs associated with asthma and allergy markers with a $P$ value less than 0.01 and MAF $>0.05$ can be found in the Supplementary Table 1 .

Supplementary data associated with this article can be found, in the online version, at https://doi.org/10.1016/j.cyto.2018.07.004.

Supplementary Table 2 provides a list of independent SNVs in RORA gene.

\subsection{Association of asthma and SPT with variants in RORA gene}

The allele A of rs2414681 and the allele C of rs7169281 were negatively associated with asthma (OR 0.70 and $\mathrm{OR}=0.69$, respectively; $\mathrm{P}<0.01$ ), the latter being also associated with non-atopic asthma

Table 3

Association between SNVs in RORA gene and asthma by logistic regression analysis adjusted for sex, age, helminth infections and ancestry.

\begin{tabular}{lcccc}
\hline SNV & OR & $99 \% \mathrm{CI}$ & P value & Perm \\
\hline Asthma & & & & \\
rs2414681 & 0.70 & $0.56-0.88$ & 0.0020 & 0.0015 \\
rs7169281 & 0.69 & $0.55-0.86$ & 0.0011 & 0.0009 \\
$\begin{array}{l}\text { Non-atopic asthma vs control } \\
\text { rs7169281 }\end{array}$ & 0.66 & $048-0.90$ & 0.0010 & 0.0009
\end{tabular}

SNV: single nucleotide variant; OR: odds ratio; $9 \%$ CI: 99\% confidence interval; Perm: permutational $\mathrm{P}$ value.
Table 4

Significant association between RORA SNVs and specific IgE by logistic regression adjusted for sex, age, helminth infections and ancestry.

\begin{tabular}{|c|c|c|c|c|}
\hline SNV & OR & $99 \% \mathrm{CI}$ & P Value & Perm \\
\hline \multicolumn{5}{|c|}{ Specific IgE for B. tropicalis } \\
\hline rs17191519 & 1.65 & $1.25-2.16$ & 0.0003 & 0.0002 \\
\hline rs17270243 & 1.44 & $1.15-1.80$ & 0.0017 & 0.0016 \\
\hline rs726914 & 1.38 & $1.11-1.72$ & 0.0035 & 0.0021 \\
\hline \multicolumn{5}{|c|}{ Specific IgE for D. pteronyssinus } \\
\hline rs10162630 & 1.43 & $1.17-1.73$ & 0.0003 & 0.0004 \\
\hline rs17191519 & 1.68 & $1.25-2.26$ & 0.0005 & 0.0004 \\
\hline rs17270243 & 1.55 & $1.21-1.98$ & 0.0005 & 0.0007 \\
\hline rs55796775 & 1.38 & $1.13-1.68$ & 0.0012 & 0.0013 \\
\hline \multicolumn{5}{|c|}{ Specific IgE for $P$. Americana } \\
\hline rs11071584 & 0.59 & $0.42-0.83$ & 0.0022 & 0.0016 \\
\hline \multicolumn{5}{|c|}{ Specific IgE for B. germanica } \\
\hline rs34720147 & 1.56 & $1.21-2.01$ & 0.0005 & 0.0005 \\
\hline rs4775301 & 1.44 & $1.12-1.84$ & 0.0045 & 0.0068 \\
\hline rs726914 & 1.70 & $1.28-2.24$ & 0.0002 & 0.0002 \\
\hline
\end{tabular}

SNV: single nucleotide variant; OR: odds ratio; $9 \%$ CI: $99 \%$ confidence interval; Perm: permutational $\mathrm{P}$ value.

Table 5

Significant associations between RORA SNVs and skin prick test (SPT) by logistic regression adjusted for sex, age, helminth infections and ancestry.

\begin{tabular}{|c|c|c|c|c|}
\hline SNV & OR & $99 \% \mathrm{CI}$ & P-value & Perm \\
\hline \multicolumn{5}{|c|}{ Skin Prick Test for Blomia tropicalis } \\
\hline rs10162630 & 1.35 & $1.11-1.64$ & 0.0026 & 0.0030 \\
\hline rs17191519 & 1.57 & $1.17-2.12$ & 0.0028 & 0.0023 \\
\hline rs17270243 & 1.47 & $1.14-1.89$ & 0.0026 & 0.0028 \\
\hline rs2414681 & 1.41 & $1.15-1.74$ & 0.0011 & 0.0012 \\
\hline rs4775301 & 1.41 & $1.14-1.74$ & 0.0013 & 0.0016 \\
\hline rs55796775 & 1.32 & $1.08-1.60$ & 0.0060 & 0.0068 \\
\hline rs7169281 & 1.39 & $1.13-1.71$ & 0.0017 & 0.0020 \\
\hline rs8024133 & 1.33 & $1.07-1.64$ & 0.0087 & 0.0107 \\
\hline \multicolumn{5}{|c|}{ Skin Prick Test for Dermatophagoides pteronyssinus } \\
\hline rs17191519 & 1.74 & $1.25-2.41$ & 0.0009 & 0.0007 \\
\hline rs4775301 & 1.42 & $1.12-1.80$ & 0.0038 & 0.0029 \\
\hline rs55796775 & 1.36 & $1.09-1.70$ & 0.0068 & 0.0058 \\
\hline rs8024133 & 1.40 & $1.10-1.78$ & 0.0058 & 0.0049 \\
\hline \multicolumn{5}{|c|}{ Skin Prick Test for Periplaneta Americana } \\
\hline rs10162630 & 1.35 & $1.07-1.71$ & 0.0106 & 0.0122 \\
\hline rs11071584 & 0.63 & $0.48-0.83$ & 0.0010 & 0.0010 \\
\hline rs12903172 & 1.53 & $1.18-1.99$ & 0.0013 & 0.0012 \\
\hline rs34720147 & 1.40 & $1.09-1.80$ & 0.0087 & 0.0132 \\
\hline rs726914 & 1.51 & $1.14-1.99$ & 0.0037 & 0.0038 \\
\hline \multicolumn{5}{|c|}{ Skin Prick Test for Blatella germanica } \\
\hline rs12903172 & 1.74 & $1.26-2.40$ & 0.0007 & 0.0004 \\
\hline rs726914 & 1.59 & $1.13-2.24$ & 0.0084 & 0.0085 \\
\hline
\end{tabular}

SNV: single nucleotide variant; OR: odds ratio; $99 \%$ CI: 99\% confidence interval; Perm: permutational $\mathrm{P}$ value.

(Table 3). Both SNVs were also positively associated with SPT for Blomia tropicalis $(\mathrm{P}<0.01)$ (Table 5).

\subsection{Association of specific IgE levels for aeroallergens with variants in RORA gene}

The polymorphisms rs10162630, rs17191519, rs17270243 and rs55796775 were positively associated with sIgE positivity for $D$. pteronyssinus (A allele, OR 1.43; G allele, OR 1.68; G allele, OR 1.55 and T allele, OR 1.38, respectively; P < 0.01, two of which rs17191519 (G allele, $\mathrm{OR} 1.65, \mathrm{P}=0.0003$ ) rs17270243 (G allele, OR 1.44, $\mathrm{P}=0.0017$ ) were also positively associated with sIgE for $B$. tropicalis. Moreover, the allele A rs726914 was positively associated with sIgE for B. tropicalis (OR 1.38, $\mathrm{P}=0.0035$ ) and $\operatorname{sIgE}$ for B. germanica (OR 1.70, $\mathrm{P}=0.0002$ ). Two other variants were positively associated with $\operatorname{sigE}$ 
for B. germanica and only the variant rs11071584 (A allele) had a negative association for $P$. americana (OR 0.59 and $\mathrm{P}=0.0017$ ) (Table 4).

\subsection{Association of skin prick test with variants in RORA gene}

Table 5 shows the association between variants in RORA and skin prick test. It was observed a statistically positive association for eight SNVs in the RORA gene with SPT for B. tropicalis (P $<0.01$ ). Of these eight, four SNVs were also positively associated with SPT for $D$. pteronyssinus $(\mathrm{P}<0.01)$. It has been demonstrated a positive association between variants rs12903172 and rs726914 with SPT for cockroaches. Similarly, the association with P. americana specific IgE rs11071584 was negatively associated with SPT for this same aeroallergen (OR 0.63; $\mathrm{P}=0.001$ ).

\subsection{Association between SNVs in RORA gene and type 2 cytokine production}

The $\mathrm{T}$ allele of rs55796775 was positively associated with IL-13 production upon $D$. pteronyssinus stimulation in peripheral blood cells (OR 1.33, CI 1.08-1.64; $\mathrm{P}=0.0072$ ). It was not possible to obtain the adjusted analysis for IL-5 production due to the small number of individuals with polymorphic alleles for SNVs in RORA gene.

It is noteworthy that rs10162630, rs17191519, rs17270243 and rs55796775 were associated with SPT and sIgE for D. pteronyssinus and/ or $B$. tropicalis, then it was evaluated the effect of presence one or two polymorphic alleles on the production of IL- 5 and IL- 13 stimulated by these mites in atopic individuals. The A alele of rs10162630 was associated with high levels of IL-13 production by Blomia tropicalis stimulus ( $\mathrm{P}=0.046$, Fig. 2A). Atopic individuals with rs17191519 AG/ GG genotypes exhibited higher IL-5 production upon stimulation with D. pteronyssinus, when compared to individuals with rs17191519 AA genotype ( $\mathrm{P}=0.045$, Fig. $2 \mathrm{~B}$ ). Regardless of other genotypes, no significant differences were observed in IL-5 and IL-13 production under stimulation with $B$. tropicalis and/or $D$. pteronyssinus among the study groups (data not shown).

\subsection{Haplotype analysis}

To perform the haplotype analysis, SNVs in high linkage disequilibrium and associated with at least four different variables. In

A

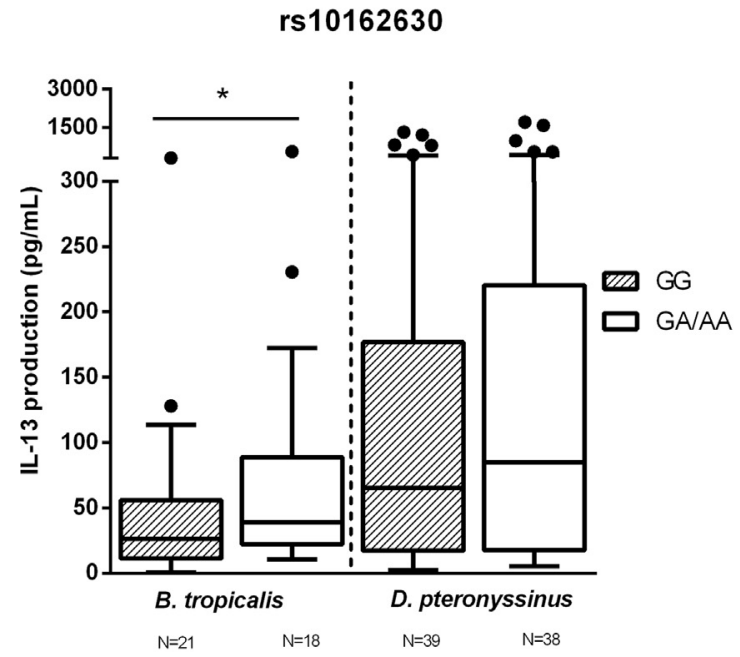

Table 6 we present the haplotype analysis for SNVs in RORA obtained our study. The SNVs rs10162630 (A allele), rs17191519 (G allele), rs17270243 (G allele) and rs55796775 (T allele) have statistically significant positive associations with two different outcomes (Tables 4 and 5). In the haplotype analysis, the positive association was observed between the haplotype AGGT and SPT and between the haplotype AGGT and sIgE, for house dust mites $(P=0.001)$. Other haplotypes of these SNVs were also associated with the SPT and sIgE allergy markers.

\subsection{In silico analysis}

Analysis from the regulomeDB showed that only the variant rs17191519 had a value equals to 3a (Table 2). For the other SNVs of this study, the values of the regulomeDB were above the 3 a value.

Using the Haploview program, the linkage disequilibrium (LD) analysis, was performed, in which three blocks were found (Fig. 3).

The differential expression of RORA in cutaneous tissue was obtained using the GTEx platform. In skin not exposed to sunlight it was found polymorphisms rs10162630 and rs55796775 significantly associated with expression of the RORA gene $(\mathrm{P}<0.05)$. While in sunexposed skin, it was found that rs17191519 and rs17270243 were also associated with the expression of RORA (P < 0.01) (Fig. 4A-D). Only variant rs17270243 was significantly associated with expression of the RORA gene in lung tissue (Fig. 4E).

\section{Discussion}

Many advances have been made to unveil, at the molecular level, the complexity of asthma and allergic diseases [33]. The RORA transcription factor has been investigated and it has been found that it participates in lung development during the fetal life and is associated with pulmonary allergic inflammation [24,34]. Studies of candidate genes and GWAS have identified the RORA gene region as involved in susceptibility to various diseases, including asthma [20,22,24]. Herein it is reported for the first time that genetic variants in RORA are significantly associated with asthma and allergy markers in an admixed population, and since atopic sensitization still remains the strongest risk factor for asthma on the western part of the planet [35], it is speculated that these variants may play a role in asthma occurrence.

In our study, four SNVs in RORA (rs10162630, rs17191519, rs17270243 and rs55796775) and their haplotypes, especially the

\section{B}

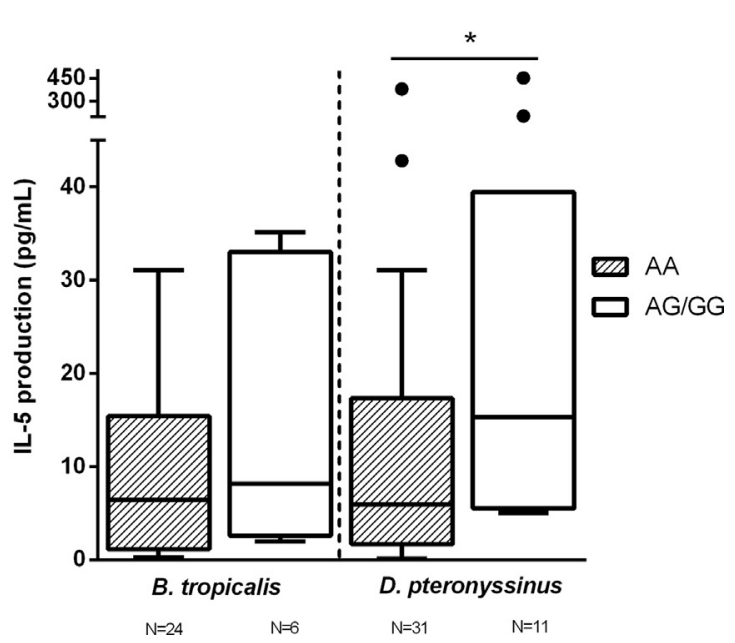

Fig. 2. IL-5 and IL-13 production by atopic individuals according to SNVs RORA genotype. (A) IL-13 production by B. tropicalis (GG $=26,56$ [11.65-55.99] pg/ml; $\mathrm{AG} / \mathrm{AA}=40.79[23.50-89.94] \mathrm{pg} / \mathrm{ml}$ ) and $D$. pteronyssinus stimulus $(\mathrm{GG}=66.67[17.45-184.70] \mathrm{pg} / \mathrm{ml} ; \mathrm{AG} / \mathrm{AA}=87.36[17.73-231.30] \mathrm{pg} / \mathrm{ml})$. (B) $\mathrm{IL}-5 \mathrm{pro}-$ duction by Blomia tropicalis $(\mathrm{AA}=6.46[1.14-15.42] \mathrm{pg} / \mathrm{ml}$; $\mathrm{AG} / \mathrm{GG}=8.14[2.58-33.01]$ ) and D. pteronyssinus stimulus $(\mathrm{AA}=5.92[1.67-17.32] \mathrm{pg} / \mathrm{ml}$; $\mathrm{AG}$ / $\mathrm{GG}=14.18[5.46-81.15])$. 
Table 6

RORA haplotypes associated with skin prick test and specific IgE for house dust mites.

\begin{tabular}{|c|c|c|c|c|c|c|c|c|}
\hline \multirow[t]{2}{*}{ Haplotype } & \multirow[t]{2}{*}{ rs10162630 } & \multirow[t]{2}{*}{ rs17191519 } & \multirow[t]{2}{*}{ rs17270243 } & \multirow[t]{2}{*}{ rs55796775 } & \multicolumn{2}{|c|}{ Frequencies } & \multirow[t]{2}{*}{$\mathrm{OR}^{\mathrm{a}}(95 \% \mathrm{CI})$} & \multirow[t]{2}{*}{ P-value } \\
\hline & & & & & Cases & Controls & & \\
\hline Reference & G & A & A & G & 0.46 & 0.37 & 1.00 & \\
\hline \multicolumn{9}{|c|}{ Haplotype association with SPT for $B$. tropicalis } \\
\hline 1SPT.B & A & A & A & $\mathrm{T}$ & 0.37 & 0.32 & $\begin{array}{l}1.44 \\
(1.12-1.84)\end{array}$ & 0.004 \\
\hline 2SPT.B & G & A & G & G & 0.06 & 0.05 & $\begin{array}{l}1.64 \\
(0.96-2.81)\end{array}$ & 0.071 \\
\hline 3SPT.B & G & G & G & G & 0.05 & 0.04 & $\begin{array}{l}1.72 \\
(0.96-3.07)\end{array}$ & 0.067 \\
\hline 4SPT.B & A & G & G & $\mathrm{T}$ & 0.07 & 0.04 & $\begin{array}{l}2.38 \\
(1.45-3.89)\end{array}$ & 0.0006 \\
\hline 5SPT.B & A & A & A & G & 0.04 & 0.04 & $\begin{array}{l}1.33 \\
(0.78-2.27)\end{array}$ & 0.03 \\
\hline 6SPT.B & A & A & G & $\mathrm{T}$ & 0.01 & 0.01 & $\begin{array}{l}1.06 \\
(0.31-3.60)\end{array}$ & 0.93 \\
\hline \multicolumn{9}{|c|}{ Haplotype association with IgE specific for $B$. tropicalis } \\
\hline 1IgE.B & A & A & A & $\mathrm{T}$ & 0.33 & 0.33 & $\begin{array}{l}1.11 \\
(0.90-1.38)\end{array}$ & 0.32 \\
\hline 2IgE.B & G & A & G & G & 0.06 & 0.05 & $\begin{array}{l}1.47 \\
(0.91-2.38)\end{array}$ & 0.12 \\
\hline 3IgE.B & G & G & G & G & 0.05 & 0.04 & $\begin{array}{l}1.53 \\
(0.92-2.55)\end{array}$ & 0.1 \\
\hline 4IgE.B & A & G & G & $\mathrm{T}$ & 0.06 & 0.04 & $\begin{array}{l}2.01 \\
(1.26-3.21)\end{array}$ & 0.004 \\
\hline 5IgE.B & A & A & A & G & 0.04 & 0.04 & $\begin{array}{l}1.14 \\
(0.71-1.83)\end{array}$ & 0.58 \\
\hline 6IgE.B & A & A & G & $\mathrm{T}$ & 0.01 & 0.02 & $\begin{array}{l}0.69 \\
(0.23-2.06)\end{array}$ & 0.51 \\
\hline \multicolumn{9}{|c|}{ Haplotype association with SPT for $D$. pteronyssinus } \\
\hline 1SPT.D & A & A & A & $\mathrm{T}$ & 0.39 & 0.32 & $\begin{array}{l}1.52 \\
(1.15-2.00)\end{array}$ & 0.003 \\
\hline 2SPT.D & G & A & G & G & 0.06 & 0.05 & $\begin{array}{l}1.61 \\
(0.90-2.86)\end{array}$ & 0.11 \\
\hline 3SPT.D & G & G & G & G & 0.06 & 0.04 & $\begin{array}{l}2.17 \\
(1.19-3.95)\end{array}$ & 0.011 \\
\hline 4SPT.D & A & A & A & G & 0.04 & 0.04 & $\begin{array}{l}1.11 \\
(0.58-2.10)\end{array}$ & 0.75 \\
\hline 5SPT.D & A & G & G & $\mathrm{T}$ & 0.07 & 0.04 & $\begin{array}{l}2.41 \\
(1.42-4.10)\end{array}$ & 0.001 \\
\hline 6SPT.D & A & A & G & $\mathrm{T}$ & 0.005 & 0.02 & $\begin{array}{l}0.39 \\
(0.05-2.89)\end{array}$ & 0.36 \\
\hline \multicolumn{9}{|c|}{ Haplotype association with IgE specific for D. pteronyssinus } \\
\hline 1IgE.D & A & A & A & $\mathrm{T}$ & 0.38 & 0.32 & $\begin{array}{l}1.55 \\
(1.21-1.98)\end{array}$ & 0.0005 \\
\hline 2IgE.D & G & A & G & G & 0.06 & 0.05 & $\begin{array}{l}1.84 \\
(1.09-3.11)\end{array}$ & 0.023 \\
\hline 3IgE.D & G & G & G & G & 0.06 & 0.04 & $\begin{array}{l}2.01 \\
(1.16-3.51)\end{array}$ & 0.014 \\
\hline 4IgE.D & A & G & G & $\mathrm{T}$ & 0.07 & 0.04 & $\begin{array}{l}2.73 \\
(1.68-4.44\end{array}$ & 0.0001 \\
\hline 5IgE.D & A & A & A & G & 0.05 & 0.04 & $\begin{array}{l}1.59 \\
(0.95-2.66)\end{array}$ & 0.08 \\
\hline 6IgE.D & A & A & G & $\mathrm{T}$ & 0.01 & 0.02 & $\begin{array}{l}1.04 \\
(0.30-3.58)\end{array}$ & 0.95 \\
\hline
\end{tabular}

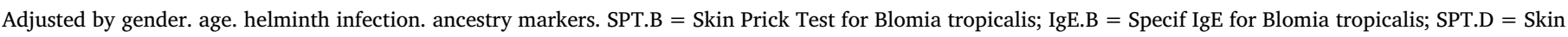
Prick Test for Dermatophagoides pteronyssinus; IgE.D = Specific IgE for Dermatophagoides pteronyssinus.

AGGT haplotype, were associated with increasing risk for atopy markers production. In silico analysis GTEx revealed that these SNVs have increased RORA expression for the polymorphic allele. In addition, rs55796775 and rs10162630 were associated with increased production of IL-13 in dust mite-stimulated cell cultures, while atopic individuals with genotype GG/AG of rs17191519 presented high levels of IL-5 production when stimulated with $D$. pteronyssinus. The transcription factor RORA is essential for ILC2 cells development that are rapid and potent producers of the type 2 cytokines Il-5 and IL-13 [11,17]. Recently, Halim et al. have proved the importance of ILC2-derived IL13 in the efficient recruitment of memory Th2 cells to the allergen- challenged lung through collaboration with lung dendritic cells $[8,36]$. Therefore, as the presence of RORA is related to increased allergic lung inflammation mainly through an immune response mediated by ILC2 cells, the genetic variants previously cited may play a role in the pathogenesis of asthma and allergic diseases $[6,37]$. However, to our knowledge, none of these variants in RORA have been associated with asthma and/or allergies in other populations so far. Probably this result is related to the type of population evaluated in this study, composed of African descendants, whereas other studies involving the RORA gene and asthma were carried out in Europeans and/or their descendants.

Two SNVs, rs2414681 and rs7169281, were associated with 


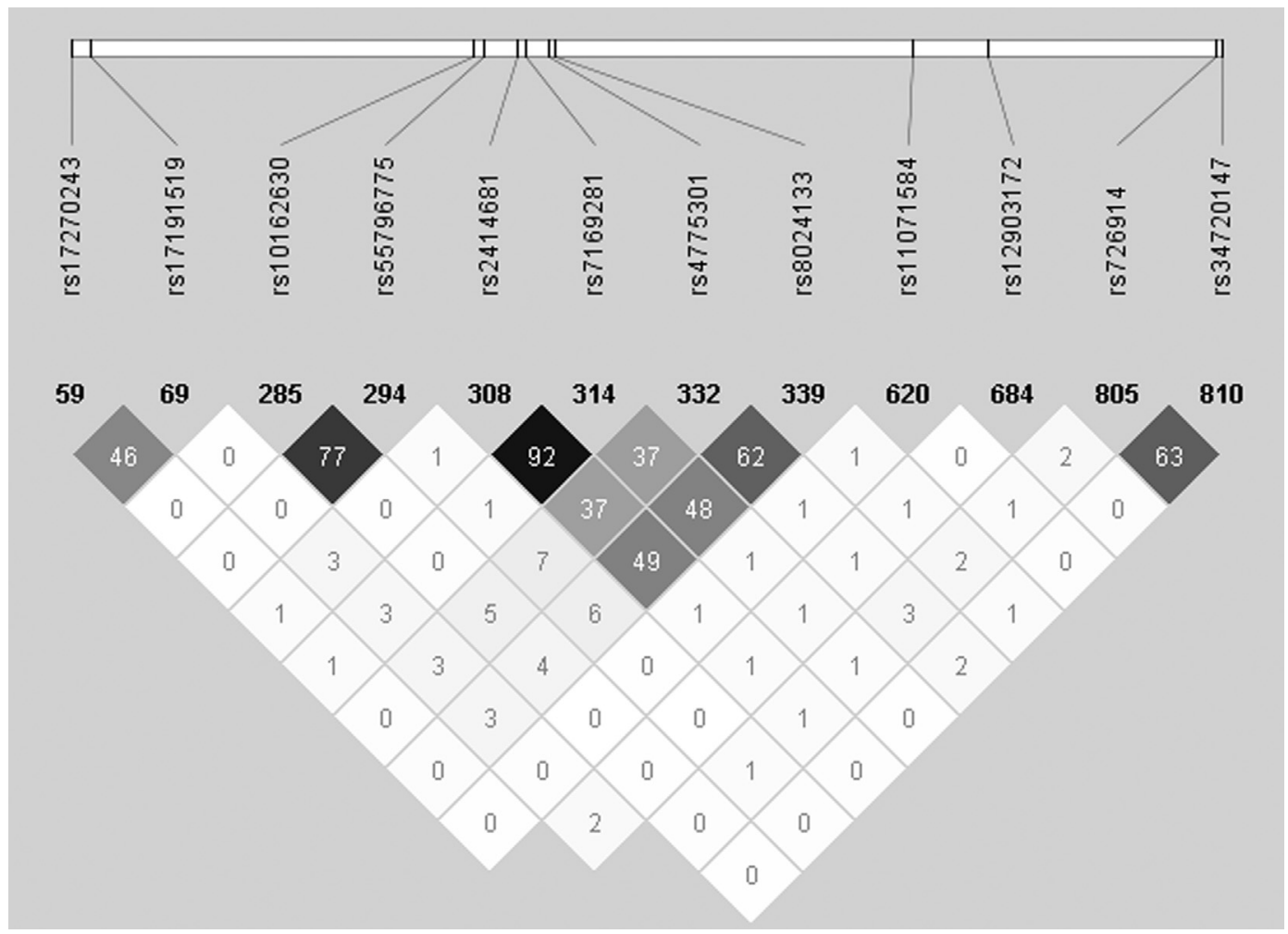

Fig. 3. Pairwise LD within Haploview using the $r$ squared statistic for the RORA gene. Intensity of shading indicates the degree of confidence in the $r$ squared value.

decreased risk for asthma and rs7169281 (allele C) was negatively associated with non-atopic asthma, suggesting that these variants could provide protection against the development of this disease phenotype. According to literature data, non-atopic asthma has independent pathogenic mechanisms of a type 2 immune response and often progresses to severe asthma with participation of Th17 and Th1 cytokines, but not type 2 cytokines [7]. On the other hand, these SNVs can trigger atopy as they were associated with SPT positivity for $B$. tropicalis, which is a risk factor for the development of allergies, such as atopic asthma [35,37].

Alleles C of rs12903172, T of rs34720147 and A of rs726914 were
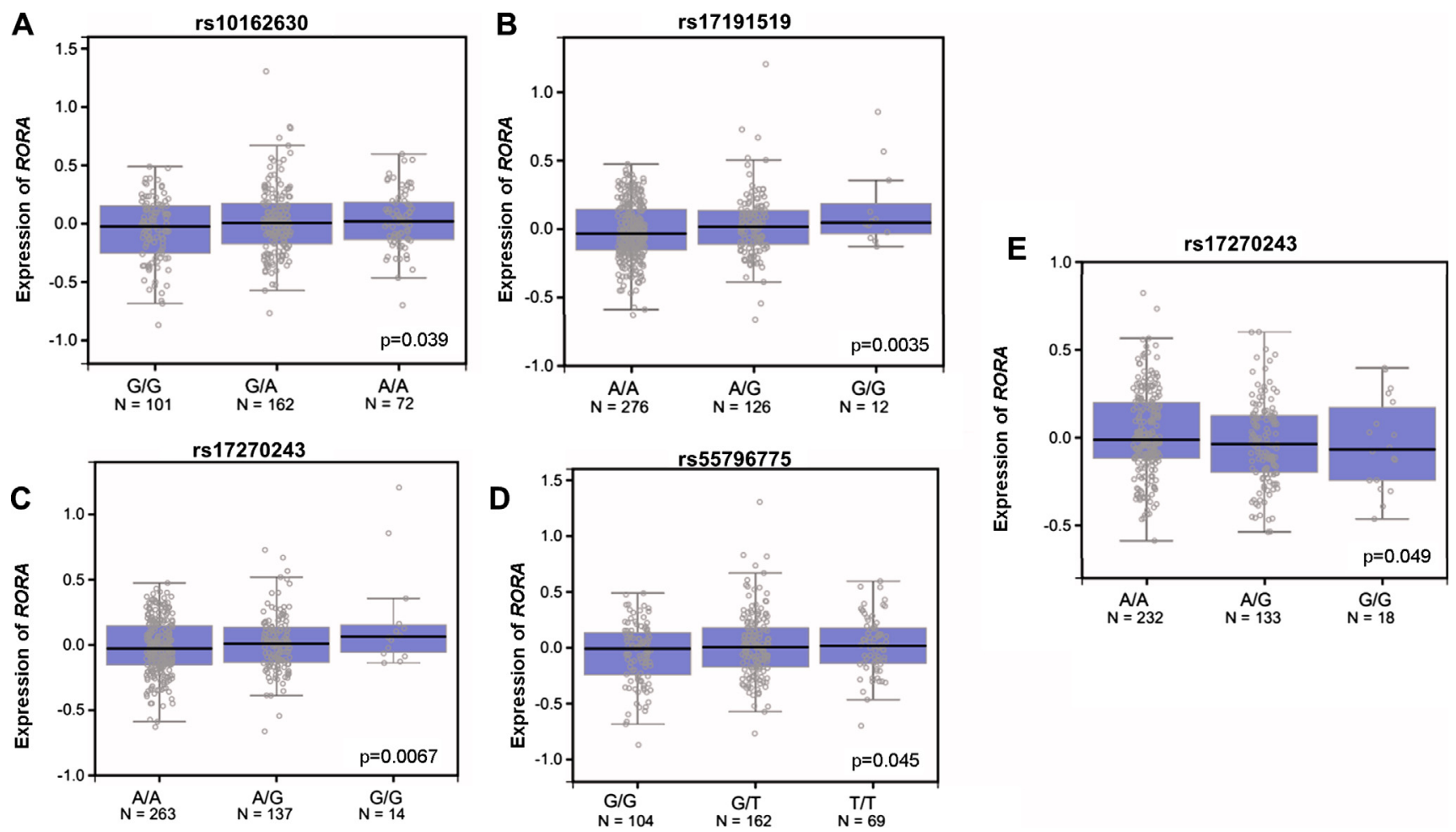

Fig. 4. GTEx-Gene expression level of RORA grouped by genotypes of (A) 10162630 from skin non-exposed to sunlight; (B) rs17191519 from sun-exposed skin; (C) rs17270243 from sun-exposed skin; (D) rs55796775 from skin non-exposed to sunlight; (E) rs17270243 from lung tissue. 
positively associated with SPT positivity and serum IgE production for cockroach allergens. Other SNVs (Allele C of rs4775301 and allele T of rs8024133) also showed association with increased allergy markers in this study. Therefore, exposure to these aeroallergens in those individuals with such polymorphisms may trigger an immune response with production of $\operatorname{sigE}$ and type 2 cytokines, leading to the emergence of allergic diseases [6]. In this work, only the rs11071584 polymorphism showed negative association with reactivity for SPT and SIgE tests, suggesting that this may be a protective marker for allergic diseases.

The LD graph shows that six of the SNVs analyzed here form three blocks in strong LD. They were associated with allergy markers indicating that the $15 \mathrm{q} 22$ region, in which RORA is found, can be responsible for occurrence of allergic diseases. All the variants presented here are in the intronic region of the RORA-1 isoform for this transcription factor, which is considered the active isoform in humans. SNVs located in regulatory parts of the intronic region may alter gene transcoding and alternative splicing, and consequently the RORA production [38]. On the other hand, the values of regulomeDB score did not present high values for the SNVs in this study and this fact is credited to the absence of known ligands for RORA.

Although some GWAS have shown association of the RORA gene region with asthma and allergic diseases $[20,22,39]$, only one candidate gene study was found in which variants in the RORA gene were significantly associated with asthma [24]. However, these variants were different from those observed in our study. In addition, unlike our findings, the same author [24] has not found association of RORA gene with atopic sensitization. It is hypothesized that these differences can be attributed to the differences in the genotyping panels used to study allelic frequencies and the different ethnicities of the studied populations. In fact, the studies carried out to date on the RORA gene involve populations of European origin, whereas in our study we dealt with admixed populations of African descendants. Literature data report notable disparities in the frequencies of susceptible alleles for asthma in African descendants [40,41].

In conclusion, individuals in our population with genetic variants in RORA have a higher risk of developing asthma and atopy and protection for non-atopic asthma. To our knowledge, this is the first work associating SNVs in the RORA gene with asthma and allergic sensitization in a population of African descendants. Studies in diverse populations are important to reveal new etiological mechanisms, involving possible gene-environment differences, as well as to examine the consistency of established associations. Future work will be necessary to understand how the loci described in this study control the disease and whether this gene could be used as a target for the treatment of allergic diseases.

\section{Acknowledgments}

To Adriele Silveira in contributing for technical support. To the team of the Laboratory of immunopharmacology and Molecular Biology (Institute of Health Sciences/UFBA) for technical assistance.

\section{Funding}

This work was supported by CNPq National Research Council Brazil and FAPESB State of Bahia, Brazil Research Council.

\section{References}

[1] S. Croisant, "Epidemiology of asthma: prevalence and burden of disease," in Heterogeneity in Asthma, Springer, 2014, pp. 17-29.

[2] I. Bara, A. Ozier, J.T. De Lara, R. Marthan, P. Berger, Pathophysiology of bronchial smooth muscle remodelling in asthma, Eur. Respir. J. 36 (5) (2010) 1174-1184.
[3] S.E. Wenzel, Asthma phenotypes: the evolution from clinical to molecular approaches, Nat. Med. 18 (5) (2012) 716-725.

[4] S.P. Doherty, J. Grabowski, C. Hoffman, S.P. Ng, J.T. Zelikoff, Early life insult from ci garette smoke may be predictive of chronic diseases later in life, Biomarkers 14 (suppl. 1) (2009) 97-101.

[5] M. Jaradat, et al., Modulatory role for retinoid-related orphan receptor $\alpha$ in allergeninduced lung inflammation, Am. J. Respir. Crit. Care Med. 174 (12) (2006) 1299-1309.

[6] J.V. Fahy, Type 2 inflammation in asthma [mdash] present in most, absent in many, Nat Rev. Immunol. 15 (1) (2015) 57-65.

[7] L. Cosmi, F. Liotta, E. Maggi, S. Romagnani, F. Annunziato, Th17 cells: new players in asthma pathogenesis, Allergy 66 (8) (2011) 989-998.

[8] T.Y. Halim, et al., Group 2 innate lymphoid cells license dendritic cells to potentiate memory TH2 cell responses, Nat. Immunol. 17 (1) (2016) 57-64.

[9] A.M. Jetten, E. Ueda, The ROR nuclear orphan receptor subfamily: critical regulators of multiple biological processes, Prog. Nucleic Acid Res. Mol. Biol. 69 (2001) 205-247.

[10] J. Mjösberg et al., "The transcription factor GATA3 is essential for the function of human type 2 innate lymphoid cells," immunity, vol. 37, no. 4, pp. 649-659, 2012.

[11] S.H. Wong, et al., Transcription factor ROR [alpha] is critical for nuocyte development, Nat. Immunol. 13 (3) (2012) 229-236.

[12] R.L. Fitzsimmons, P. Lau, G.E. Muscat, Retinoid-related orphan receptor alpha and the regulation of lipid homeostasis, J. Steroid Biochem. Mol. Biol. 130 (3) (2012) 159-168.

[13] L.A. Solt, T.P. Burris, Action of RORs and their ligands in (patho) physiology, Trends Endocrinol. Metab. 23 (12) (2012) 619-627.

[14] D.J. Mangelsdorf, R.M. Evans, The RXR heterodimers and orphan receptors, Cell 83 (6) (1995) 841-850.

[15] D. N. Cook, H. S. Kang, and A. M. Jetten, "Retinoic acid-related orphan receptors (RORs): regulatory functions in immunity, development, circadian rhythm, and metabolism," Nucl. Recept. Res., vol. 2, 2015.

[16] H. Duez, B. Staels, Nuclear receptors linking circadian rhythms and cardiometabolic control, Arterioscler. Thromb. Vasc. Biol. 30 (8) (2010) 1529-1534.

[17] T.Y. Halim, R.H. Krauß, A.C. Sun, F. Takei, Lung natural helper cells are a critical source of Th2 cell-type cytokines in protease allergen-induced airway inflammation, Immunity 36 (3) (2012) 451-463.

[18] W. Cookson, The alliance of genes and environment in asthma and allergy, Nature 402 (1999) 5-11.

[19] S.T. Holgate, Genetic and environmental interaction in allergy and asthma, J. Allergy Clin. Immunol. 104 (6) (1999) 1139-1146.

[20] G.N. Costa, et al., A genome-wide association study of asthma symptoms in Latin American children, BMC Genet. 16 (1) (2015) 141.

[21] M. Imboden, et al., Genome-wide association study of lung function decline in adults with and without asthma, J. Allergy Clin. Immunol. 129 (5) (2012) 1218-1228.

[22] M.F. Moffatt, et al., A large-scale, consortium-based genomewide association study of asthma, N. Engl. J. Med. 363 (13) (2010) 1211-1221.

[23] B.D. Spycher, et al., Genome-wide prediction of childhood asthma and related phenotypes in a longitudinal birth cohort, J. Allergy Clin. Immunol. 130 (2) (2012) 503-509.

[24] N. Acevedo, et al., Interaction between retinoid acid receptor-related orphan receptor alpha (RORA) and neuropeptide S receptor 1 (NPSR1) in asthma, PloS One 8 (4) (2013) e60111.

[25] N.M. Alcantara-Neves, et al., The effect of single and multiple infections on atopy and wheezing in children, J. Allergy Clin. Immunol. 129 (2) (2012) 359-367.

[26] M.L. Barreto, et al., Risk factors and immunological pathways for asthma and other allergic diseases in children: background and methodology of a longitudinal study in a large urban center in Northeastern Brazil (Salvador-SCAALA study), BMC Pulm. Med. 6 (1) (2006) 15.

[27] C.A. Figueiredo, et al., Chronic intestinal helminth infections are associated with immune hyporesponsiveness and induction of a regulatory network, Infect. Immun. 78 (7) (2010) 3160-3167.

[28] F.S. Kehdy, et al., Origin and dynamics of admixture in Brazilians and its effect on the pattern of deleterious mutations, Proc. Natl. Acad. Sci. 112 (28) (2015) 8696-8701.

[29] S. Purcell, et al., PLINK: a tool set for whole-genome association and population-based linkage analyses, Am. J. Hum. Genet. 81 (3) (2007) 559-575.

[30] G.A. Queiroz, et al., IL33 and IL1RL1 variants are associated with asthma and atopy in a Brazilian population, Int. J. Immunogenet. 44 (2) (2017) 51-61.

[31] Y. Wang, L.P. Zhao, S. Dudoit, A fine-scale linkage-disequilibrium measure based on length of haplotype sharing, Am. J. Hum. Genet. 78 (4) (2006) 615-628.

[32] A.P. Boyle, et al., Annotation of functional variation in personal genomes using RegulomeDB, Genome Res. 22 (9) (2012) 1790-1797.

[33] S. Bunyavanich, E.E. Schadt, Systems biology of asthma and allergic diseases: a multiscale approach, J. Allergy Clin. Immunol. 135 (1) (2015) 31-42.

[34] E. Melén, et al., Genome-wide association study of body mass index in 23000 individuals with and without asthma, Clin. Exp. Allergy 43 (4) (2013) 463-474.

[35] A. Simpson, et al., Beyond atopy: multiple patterns of sensitization in relation to asthma in a birth cohort study, Am. J. Respir. Crit. Care Med. 181 (11) (2010) 1200-1206.

[36] T.Y. Halim, et al., Group 2 innate lymphoid cells are critical for the initiation of adaptive T helper 2 cell-mediated allergic lung inflammation, Immunity 40 (3) (2014) 425-435.

[37] B.N. Lambrecht, H. Hammad, The airway epithelium in asthma, Nat. Med. 18 (5) (2012) 684-692.

[38] N. Hubner, et al., Integrated transcriptional profiling and linkage analysis for identification of genes underlying disease, Nat. Genet. 37 (3) (2005) 243-253.

[39] A. Ramasamy, et al., Genome-wide association studies of asthma in population-based cohorts confirm known and suggested loci and identify an additional association near HLA, Plos One 7 (9) (2012) e44008.

[40] R.A. Mathias, et al., A genome-wide association study on African-ancestry populations for asthma, J. Allergy Clin. Immunol. 125 (2) (2010) 336-346.

[41] C. Vergara, et al., African ancestry is a risk factor for asthma and high total IgE levels in African admixed populations, Genet. Epidemiol. 37 (4) (2013) 393-401. 